\title{
Heavy flavour corrections to polarised and unpolarised deep-inelastic scattering at 3-loop order*
}

\author{
J. Ablinger ${ }^{a}$, A. Behring ${ }^{\dagger b, c}$, J. Blümlein ${ }^{b}$, A. De Freitas ${ }^{b}$, A. Hasselhuhn ${ }^{d}$, \\ A. von Manteuffel ${ }^{e, f}$, M. Round ${ }^{a}$, C. Schneider ${ }^{a}$, F. Wißbrock ${ }^{g}$ \\ ${ }^{a}$ Research Institute for Symbolic Computation (RISC), Johannes Kepler University, \\ Altenbergerstraße 69, A-4040, Linz, Austria \\ ${ }^{b}$ Deutsches Elektronen-Synchrotron, DESY, Platanenallee 6, D-15738 Zeuthen, Germany \\ ${ }^{c}$ Institut für Theoretische Teilchenphysik und Kosmologie, RWTH Aachen University, \\ D-52056 Aachen, Germany \\ ${ }^{d}$ Institut für Theoretische Teilchenphysik, Karlsruher Institut für Technologie (KIT), \\ D-76128 Karlsruhe, Germany \\ ${ }^{e}$ PRISMA Cluster of Excellence, Institute of Physics, J. Gutenberg University, D-55099 Mainz, \\ Germany \\ ${ }^{f}$ Department of Physics and Astronomy, Michigan State University, East Lansing, MI 48824, USA \\ ${ }^{g}$ IHES, 35 Route de Chartres, F-91440 Bures-sur-Yvette, France \\ E-mail: arnd.behring@desy.de
}

\begin{abstract}
We report on progress in the calculation of 3-loop corrections to the deep-inelastic structure functions from massive quarks in the asymptotic region of large momentum transfer $Q^{2}$. Recently completed results allow us to obtain the $O\left(a_{s}^{3}\right)$ contributions to several heavy flavour Wilson coefficients which enter both polarised and unpolarised structure functions for lepton-nucleon scattering. In particular, we obtain the non-singlet contributions to the unpolarised structure functions $F_{2}\left(x, Q^{2}\right)$ and $x F_{3}\left(x, Q^{2}\right)$ and the polarised structure function $g_{1}\left(x, Q^{2}\right)$. From these results we also obtain the heavy flavour contributions to the Gross-Llewellyn-Smith and the Bjorken sum rules.
\end{abstract}

QCD Evolution 2016

May 30-June 03, 2016

National Institute for Subatomic Physics (Nikhef) in Amsterdam

${ }^{*}$ This work was supported in part by the Austrian Science Fund (FWF) grant SFB F50 (F5009-N15), the European Commission through contract PITN-GA-2012-316704 (HIGGSTOOLS) and by FP7 ERC Starting Grant 257638 PAGAP.

†Speaker. 


\section{Introduction}

Deep-inelastic scattering provides a valuable way to both test the theory of quantum chromodynamics $(\mathrm{QCD})$ and to extract theory parameters from experiments. Among these are in particular the strong coupling constant $\alpha_{s}$ [1], the parton distribution functions (PDFs) [2-4] and the masses of the charm and bottom quarks $[5,6]$. To harness the full potential of the experimental data, it is necessary to have predictions at hand for which the theoretical uncertainties keep up with the experimental accuracy. Currently, the corrections from massive quarks are still missing for a complete next-to-next-to-leading order (NNLO) analysis of the deep-inelastic scattering World data. They can be calculated analytically at NNLO in the kinematic limit $Q^{2} \gg m^{2}$ [7], where $Q^{2}$ is the virtuality of the electro-weak gauge boson and $m$ is the mass of the heavy quark. In this paper, we report on progress in the calculation of these heavy flavour corrections.

In Section 2 we describe the framework for the heavy flavour corrections to deep-inelastic scattering in the limit $Q^{2} \gg m^{2}$. In this limit, the massive operator matrix elements of the light-cone operators are the key quantities which have not been completely computed yet. Thus, we sketch the steps involved in their calculation. Section 3 contains several applications of the results which have been obtained so far. In particular, we illustrate the impact of the heavy flavour Wilson coefficients on the structure function $F_{2}\left(x, Q^{2}\right), g_{1}\left(x, Q^{2}\right)$ and $x F_{3}\left(x, Q^{2}\right)$, as well as their consequences for the polarised Bjorken sum rule and the Gross-Llewellyn-Smith sum rule. Finally, we comment on our results for the massive operator matrix element of the non-singlet operator for transversity in Section 4 and conclude in Section 5

\section{Framework of calculation}

The structure functions of deep-inelastic scattering can in general be written as convolutions of PDFs and Wilson coefficients (cf., e.g., [8]). The Wilson coefficients carry the process-dependent information about the particular scattering process at hand and can be calculated in perturbation theory. They receive contributions from massless quarks and gluons as well as from massive quarks. In the following, we will refer to the contributions of a single massive quark species (i.e. charm or bottom quarks) as the heavy flavour contributions. Starting at 3-loop order, there are also contributions from diagrams with two different massive quarks. Their calculation poses quite different challenges and is discussed elsewhere [9]. The heavy flavour contributions to the structure function $F_{2}\left(x, Q^{2}\right)$, for example, can be written as $[7,10,11]$

$$
\begin{aligned}
& F_{2}^{\mathrm{h}}\left(x, N_{F}+1, Q^{2}, m^{2}\right)= \\
& \quad x\left\{\sum _ { k = 1 } ^ { N _ { F } } e _ { k } ^ { 2 } \left[L_{q, 2}^{\mathrm{NS}}\left(x, N_{F}+1, \frac{Q^{2}}{\mu^{2}}, \frac{m^{2}}{\mu^{2}}\right) \otimes\left[f_{k}\left(x, \mu^{2}, N_{F}\right)+\bar{f}_{k}\left(x, \mu^{2}, N_{F}\right)\right]\right.\right. \\
& \quad+\frac{1}{N_{F}} L_{q, 2}^{\mathrm{PS}}\left(x, N_{F}+1, \frac{Q^{2}}{\mu^{2}}, \frac{m^{2}}{\mu^{2}}\right) \otimes \Sigma\left(x, \mu^{2}, N_{F}\right) \\
& \left.\quad+\frac{1}{N_{F}} L_{g, 2}^{\mathrm{S}}\left(x, N_{F}+1, \frac{Q^{2}}{\mu^{2}}, \frac{m^{2}}{\mu^{2}}\right) \otimes G\left(x, \mu^{2}, N_{F}\right)\right]
\end{aligned}
$$




$$
\begin{aligned}
& +e_{Q}^{2}\left[H_{q, 2}^{\mathrm{PS}}\left(x, N_{F}+1, \frac{Q^{2}}{\mu^{2}}, \frac{m^{2}}{\mu^{2}}\right) \otimes \Sigma\left(x, \mu^{2}, N_{F}\right)\right. \\
& \left.\left.+H_{g, 2}^{\mathrm{S}}\left(x, N_{F}+1, \frac{Q^{2}}{\mu^{2}}, \frac{m^{2}}{\mu^{2}}\right) \otimes G\left(x, \mu^{2}, N_{F}\right)\right]\right\} .
\end{aligned}
$$

Here, $f_{k}, \bar{f}_{k}$ and $G$ refer to the PDFs for quarks and anti-quarks of flavour $k$ and the gluon PDF, respectively. The singlet PDF combination is defined as $\Sigma=\sum_{k=1}^{N_{F}}\left(f_{k}+\bar{f}_{k}\right)$ and $\mu^{2}$ is the factorisation scale. The number of light quark flavours is denoted by $N_{F}$ and $e_{k}$ and $e_{Q}$ represent the charges of the light and heavy quarks. The heavy flavour Wilson coefficients are denoted by $L_{i, a}$ and $H_{i, a}$, where the $L$ and $H$ distinguish the cases where the electro-weak gauge boson couples to a light or a heavy quark, respectively, and the subscripts $i$ and $a$ label the initial state parton $(q, g)$ and the structure function under consideration. The symbol $\otimes$ represents the Mellin convolution, which turns into a simple product of moments if we apply to it a Mellin transformation

$$
M[f](N)=\int_{0}^{1} \mathrm{~d} x x^{N-1} f(x) .
$$

This introduces the Mellin variable $N$, to which we will refer in several places in the following.

The massless Wilson coefficients have been calculated up to 3-loop order [12], while the massive ones are available only semi-numerically up to 2-loop order [13]. ${ }^{1}$ However, it was observed in [7] that the heavy-flavour Wilson coefficients factorise into the massless Wilson coefficients and massive operator matrix elements (OME) in the kinematic limit where $Q^{2} \gg m^{2}$. In this limit, the heavy flavour Wilson coefficients $\mathscr{C}_{i, a}^{\text {asymp }}$ can be written schematically as [7]

$$
\mathscr{C}_{i, a}^{\text {asymp }}\left(x, N_{F}+1, \frac{Q^{2}}{\mu^{2}}, \frac{m^{2}}{\mu^{2}}\right)=\sum_{j} C_{j, a}\left(x, N_{F}+1, \frac{Q^{2}}{\mu^{2}}\right) \otimes A_{i j, Q}\left(x, N_{F}+1, \frac{m^{2}}{\mu^{2}}\right)+\mathscr{O}\left(\frac{m^{2}}{Q^{2}}\right) .
$$

The massless Wilson coefficients $C_{i, a}$ are evaluated for $N_{F}+1$ massless flavours and the massive OMEs $A_{i j, Q}$ are calculated with $N_{F}$ massless and one massive quark. More details on the formalism can be found in $[7,15,10,11]$. Below, we will frequently refer to the expansion coefficients of the OMEs in powers of $a_{s}=\frac{\alpha_{s}}{4 \pi}$, where the coefficient of $a_{s}^{k}$ is denoted by $A_{i j, Q}^{(k)}$.

The massive OMEs are known analytically up to 2-loop order $[7,16,15,17]$ including linear terms in the dimensional regulator $\varepsilon=D-4$, where $D$ is the dimensionality of space-time in dimensional regularisation [18]. The extension of these results to 3-loop order is the topic of our project and here we report on progress in this regard.

The massive OMEs can be extracted from calculating two-point functions with external onshell partons and operators, which introduce additional Feynman rules beyond those of QCD. Our calculation follows a diagrammatic approach, where all relevant diagrams are generated using QGRAF [19]. After inserting the Feynman rules, we simplify the colour and Dirac algebra using FORM [20] and color.h [21]. In this way, we express the diagrams in terms of roughly $10^{5}$ scalar loop integrals. In order to compute those, we first reduce them to a smaller number of master integrals using integration-by-parts relations [22, 23]. For this task we use the program Reduze 2 $[24] .^{2}$ A major task is then to actually calculate the master integrals. Over the years a number of

\footnotetext{
${ }^{1}$ For a precise implementation in Mellin space see [14].

${ }^{2}$ Reduze 2 uses the packages GiNaC [25] and Fermat [26].
} 
techniques have proven to be very useful for this task:

- Higher hypergeometric functions [27]: After deriving a Feynman parameter representation for the loop integrals, they can sometimes be brought in a form which can be integrated in terms of generalised hypergeometric $\left({ }_{p} F_{q}\right)$ or Appell functions that have convergent series representations. If this is the case, we can expand them in $\varepsilon$ and the resulting sum representations can be simplified using the summation algorithms [28, 29] implemented in Sigma [28, 30, 31], EvaluateMultiSums and SumProduction [32] with support from Harmonic.Sums [33-38] for dealing with the nested sums that arise.

- Mellin-Barnes integrals [39, 40]: Even if a direct evaluation of the Feynman parameter integrals in terms of hypergeometric functions is not possible, it may still be feasible to derive a sum representation that can be simplified using Sigma and related packages: We split up suitably formed sums of Feynman parameters at the cost of complex contour integrals [40, 41]. Afterwards, the Feynman parameter integrals can usually be done in terms of Beta and Gamma functions, while the contour integrals give rise to infinite sums via the residue theorem.

- Almkvist-Zeilberger algorithm [42,34]: If it is possible to confine the Mellin variable $N$ in the integrand of the Feynman parameter integrals to only one or a small number of places, it can be advantageous to employ the multi-variable Almkvist-Zeilberger algorithm, implemented in the package MultiIntegrate [34]. It allows to derive recurrence relations for the integrals which can subsequently be solved using the algorithms implemented in Sigma.

- Differential equations and difference equations [43, 44]: Based on the integration-by-parts relations it is possible to derive coupled systems of differential equations for the master integrals. We translate these into coupled systems of difference equations and uncouple them using Zürcher's algorithm [45], which is implemented in OreSys [46]. Once suitable initial conditions are available (e.g. from direct calculations using other methods), the difference equations can be solved using the package SolveCoupledSystem [47].

More details on the application of these techniques to the calculation of massive OMEs can be found in [44, 48-53]. The results obtained so far for the OMEs can be expressed in terms of nested sums. In particular harmonic sums [54], generalised harmonic sums [55, 36], cyclotomic sums [35] and binomially weighted sums [56,37] appear both in the intermediate steps and in the results. These structures are related to corresponding iterated integrals $[57,55,36,35,37]$ via an inverse Mellin transformation. Finally, the results for the master integrals can be inserted into the diagrams, yielding the unrenormalised expressions for the operator matrix elements.

The renormalisation procedure for the massive OMEs at $\mathscr{O}\left(a_{s}^{3}\right)$ was worked out in [10]. Since we calculate matrix elements of the local light-cone operators, it comes at no surprise that their renormalisation involves the anomalous dimensions of the operators. Using known results for the beta function, mass anomalous dimensions and lower order OMEs, we can use the pole terms of our 3-loop results to calculate the $N_{F}$-dependent part of the anomalous dimensions. 


\section{Results for structure functions}

Over the course of the recent years, a number of analytic results for the operator matrix elements have been completed. In particular, the OMEs $A_{q g, Q}^{(3)}[48], A_{g q, Q}^{(3)}[51], A_{q q, Q}^{\mathrm{PS},(3)}[48], A_{Q q}^{\mathrm{PS},(3)}$ [58], $A_{q q, Q}^{\mathrm{NS},(3)}$ [59] have been calculated. Moreover, the gluonic OME $A_{g g, Q}^{(3)}$ is known for even values of the Mellin variable $N$ [60]. These results allow for a numerical illustration of their impact on the heavy flavour Wilson coefficients of different structure functions. For the structure function $F_{2}\left(x, Q^{2}\right)$ there are five different Wilson coefficients (see Eq. (2.1)), each of which requires the knowledge of the 3-loop term of one OME. Since $A_{Q g}^{(3)}$ is not yet fully known, the Wilson coefficient $H_{g, 2}$ cannot be given yet at 3-loop order at this point. Nevertheless, we can illustrate the impact of the remaining Wilson coefficients, supplemented by the contribution of $H_{g, 2}$ up to 2-loop order for comparison. Figure 1 shows the heavy flavour contributions to $F_{2}\left(x, Q^{2}\right)$ for a fixed value of

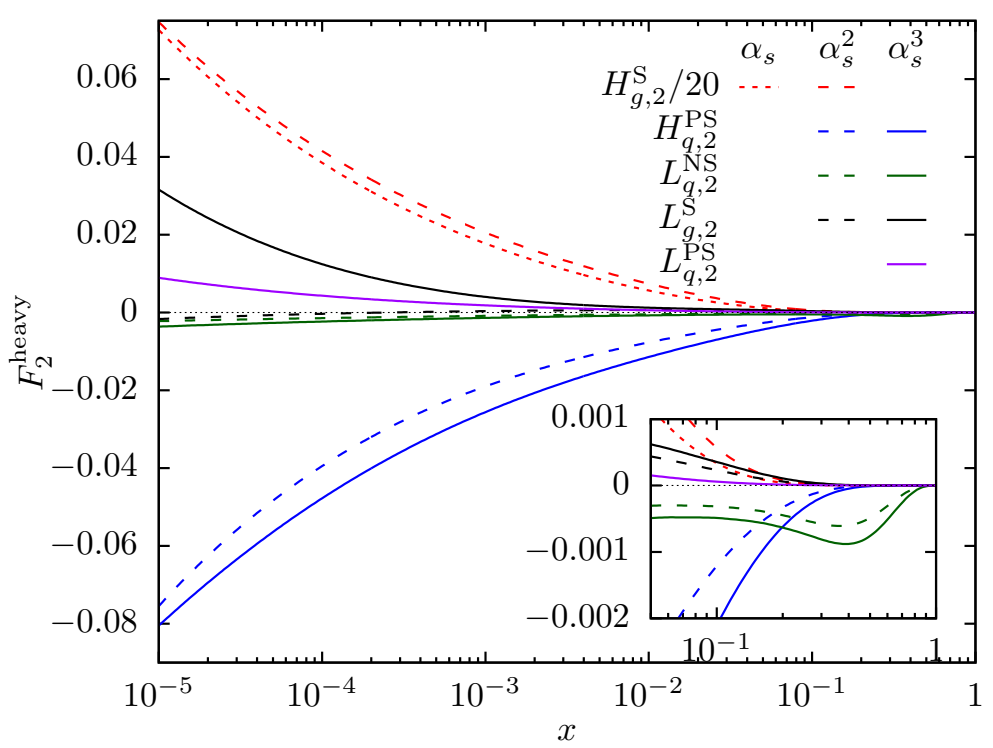

Figure 1: Illustration of the contributions to the structure function $F_{2}\left(x, Q^{2}\right)$ from the individual Wilson coefficients. The plot shows the values for $Q^{2}=100 \mathrm{GeV}^{2}$ and the scale choice $\mu^{2}=Q^{2}$ with PDFs from [2]. The charm quark mass is $m_{c}=1.59 \mathrm{GeV}^{2}$ in the on-shell scheme [5]. The inset enlarges the region of $0.05 \leq x \leq 1$.

$Q^{2}=100 \mathrm{GeV}^{2}$. The biggest contribution comes from $H_{g, 2}$. Contributions to this Wilson coefficient start at $\mathscr{O}\left(a_{s}\right)$ and are the only contribution at that order. Due to this and the fact that it involves the gluon PDF, it is quite large in the small- $x$ region and we have scaled down the curve by a factor 20 . The second largest contribution in the small- $x$ region is the pure-singlet Wilson coefficient $H_{q, 2}^{\mathrm{PS}}$. It starts at $\mathscr{O}\left(a_{s}^{2}\right)$ and is negative, except for very large values of $x$ (not visible in the plot). The large- $x$ region is dominated by the non-singlet Wilson coefficient, which also starts at $\mathscr{O}\left(a_{s}^{2}\right)$ and is negative throughout the whole $x$-range. Here, the even moments of the non-singlet $\mathrm{OME} A_{q q, Q}^{\mathrm{NS},(3)}$ enter. Somewhat smaller contributions arise from the gluon- and quark-initiated singlet Wilson coefficients $L_{g, 2}^{\mathrm{S}}$ and $L_{q, 2}^{\mathrm{PS}}$, which start at $\mathscr{O}\left(a_{s}^{2}\right)$ and $\mathscr{O}\left(a_{s}^{3}\right)$, respectively.

The non-singlet $\mathrm{OME} A_{q q, Q}^{\mathrm{NS},(3)}$ was calculated also for odd moments in [59]. This allows for applications to other structure functions besides $F_{2}\left(x, Q^{2}\right)$ : In particular the non-singlet contribution 

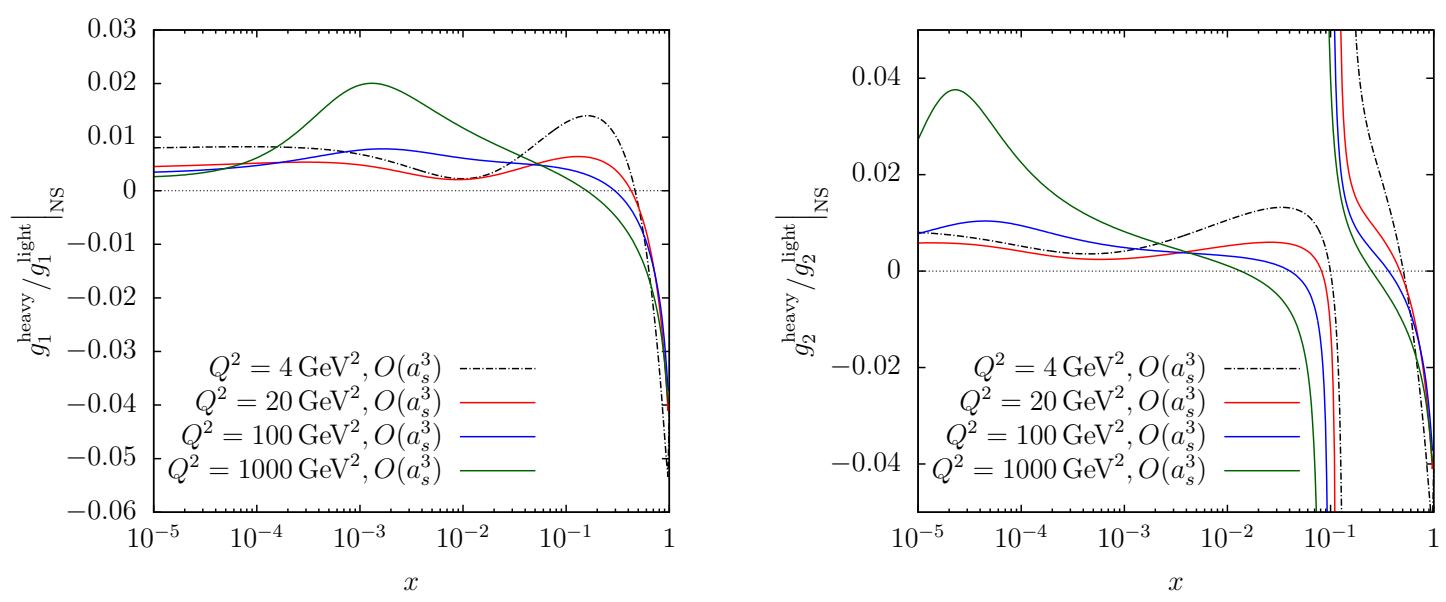

Figure 2: Ratio of the heavy over the light flavour contributions to the polarised structure functions $g_{1}\left(x, Q^{2}\right)$ (left panel) and $g_{2}\left(x, Q^{2}\right)$ (right panel) for different values of $Q^{2}$. Here, the PDFs from [61] were used; plots from [62].

to the polarised structure function $g_{1}\left(x, Q^{2}\right)$ was explored in [62]. Figure 2 illustrates the impact of the charm quarks on this structure function. Compared to the contributions from massless quarks and gluons, the charm quark constitutes around $+1 \%$ to $2 \%$ to $-5 \%$ of the non-singlet structure function. This is below the current experimental accuracy but may be of interest at future highluminosity colliders [63].

At the level of twist 2, the structure function $g_{2}\left(x, Q^{2}\right)$ is also related to $g_{1}\left(x, Q^{2}\right)$ by the Wandzura-Wilczek relation [64],

$$
g_{2}\left(x, Q^{2}\right)=-g_{1}\left(x, Q^{2}\right)+\int_{x}^{1} \frac{\mathrm{d} y}{y} g_{1}\left(y, Q^{2}\right) .
$$

This allows us to show also the impact of the charm quarks there. As can be seen in the right panel of Figure 2, the heavy quark contributions are about $1 \%$ to $4 \%$ the size of the massless contribution. The pole in the plot is due to a change of sign of $g_{2}^{\text {light }}$.

Another application of the non-singlet OME is the polarised Bjorken sum rule [65]. It is defined as the difference of the first moments of $g_{1}\left(x, Q^{2}\right)$ in electron-proton and electron-neutron scattering,

$$
\int_{0}^{1} \mathrm{~d} x\left[g_{1}^{e p}\left(x, Q^{2}\right)-g_{1}^{e n}\left(x, Q^{2}\right)\right]=\frac{1}{6}\left|\frac{g_{A}}{g_{V}}\right| C_{\mathrm{pBj}}\left(a_{S}\right),
$$

where $g_{A}$ and $g_{V}$ denote the axial-vector and vector decay constants. The perturbative coefficient $C_{\mathrm{pBj}}$ arises from first moment of the Wilson coefficients. For the massless contributions it has been calculated up to $\mathscr{O}\left(a_{s}^{4}\right)$ [66-68]. Our result for the non-singlet heavy flavour Wilson coefficient leads us to the conclusion that in the limit $Q^{2} \gg m^{2}$ the polarised Bjorken sum rule for $N_{F}$ massless quarks and one massive quark is given completely by the massless contributions for $N_{F}+1$ quarks: The massive non-singlet OME, which could modify the sum rule compared to the completely massless case, has a vanishing first moment due to fermion number conservation. However, away 

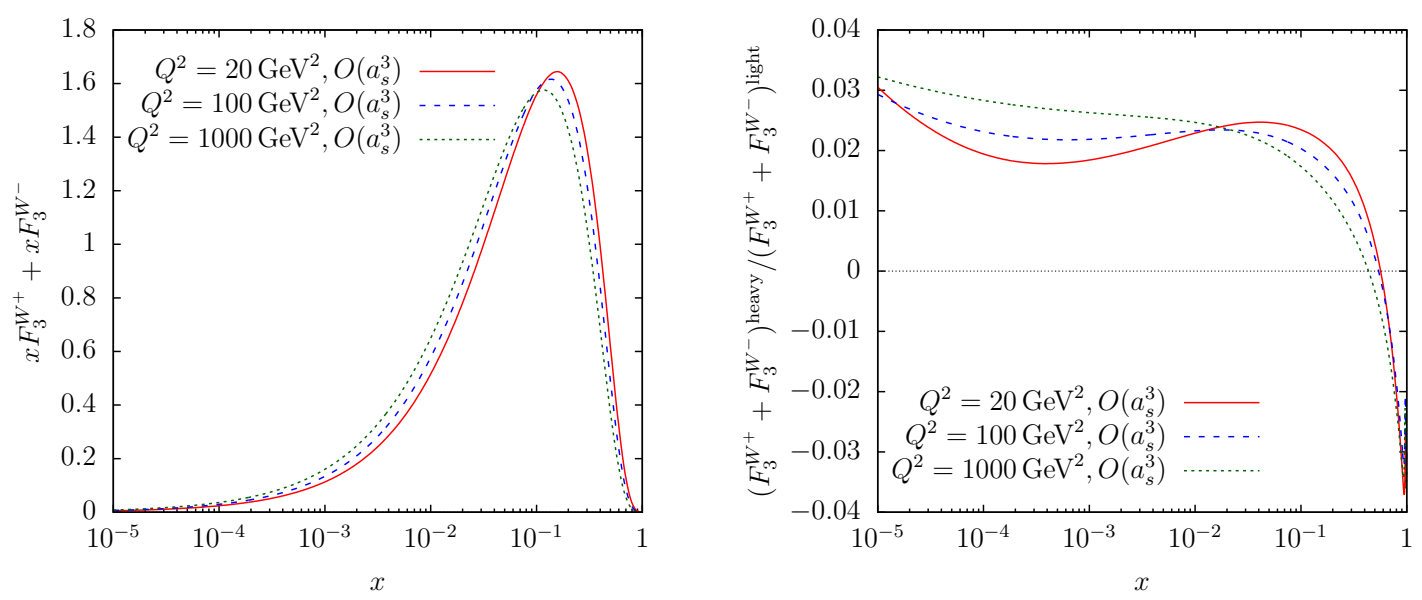

Figure 3: Left panel: Illustration of the structure function combination $x F_{3}^{W^{+}-W^{-}}\left(x, Q^{2}\right)$ including the contributions from a massive charm quark with a mass of $m_{c}=1.59 \mathrm{GeV}^{2}$ in the on-shell scheme [5]. Right panel: Ratio of the heavy quark contributions over the massless part of the same structure function; plots from [70].

from the limit $Q^{2} \gg m^{2}$, the behaviour described above no longer holds and genuine heavy flavour contributions to the polarised Bjorken sum rule arise, cf. [69].

Moreover, the same non-singlet OME enters also the charged-current structure function combination

$$
x F_{3}^{W^{+}-W^{-}}\left(x, Q^{2}\right)=x F_{3}^{W^{+}}\left(x, Q^{2}\right)+x F_{3}^{W^{-}}\left(x, Q^{2}\right) .
$$

For an explanation of the notation we refer to [71, 70]. This structure function receives contributions from two non-singlet Wilson coefficients: On the one hand, there is $L_{q, 3}^{\mathrm{NS}}$, which describes reactions in which the $W$ boson couples to a light quark and mediates a flavour transition between light quark species. This is analogous to the case of photon exchange, except for the flavour change. On the other hand, there is $H_{q, 3}^{\mathrm{NS}}$, which describes flavour excitation reactions (e.g. $s \rightarrow c$ ). Here, the $W$ boson couples to a heavy quark. This part has no analogy in the photon-mediated case. The impact of both Wilson coefficients on $x F_{3}^{W^{+}-W^{-}}\left(x, Q^{2}\right)$ is illustrated in Figure 3. The size of the heavy quark contribution is again of the order of about $3 \%$. Also in the charged current sector, there is a sum rule arising from the first moment of the structure functions: The Gross-Llewellyn-Smith sum rule [72] is given by

$$
\int_{0}^{1} \mathrm{~d} x\left[F_{3}^{\bar{v} p}\left(x, Q^{2}\right)+F_{3}^{v p}\left(x, Q^{2}\right)\right]=6 C_{\mathrm{GLS}}\left(a_{s}\right) .
$$

The massless QCD corrections to $C_{\mathrm{GLS}}$ are again known to $\mathscr{O}\left(a_{s}^{4}\right)[66,73,74]$ and the situation in the heavy quark sector is similar to the polarised case: Due to the vanishing first moment of $A_{q q, Q}^{\mathrm{NS}}$, the influence of the heavy quark in the asymptotic region $Q^{2} \gg m^{2}$ reduces to incrementing the number of massless flavours $\left(N_{F} \rightarrow N_{F}+1\right)$. For the power corrections in $m^{2} / Q^{2}$ up to 2-loop order, we refer to [69]. 


\section{Operator matrix element for transversity}

The non-singlet results discussed so far involve the flavour non-singlet vector or axial-vector operators. A very similar calculation can be performed for the non-singlet tensor operator

$$
O_{q, r}^{\mathrm{TR}, \mathrm{NS}, \mu \mu_{1} \ldots \mu_{N}}(z)=\mathrm{i}^{N-1} S\left[\bar{\psi}(z) \sigma^{\mu \mu_{1}} D^{\mu_{2}} \ldots D^{\mu_{N}} \frac{\lambda_{r}}{2} \psi(z)\right]-\text { trace terms },
$$

where $D^{\mu}$ is the covariant derivative, $\psi$ is the quark field operator, $\lambda_{r}$ denotes the Gell-Mann matrices of $\mathrm{SU}(3)_{\text {flavour }}, \sigma^{\mu \nu}=\frac{\mathrm{i}}{2}\left(\gamma^{\mu} \gamma^{\nu}-\gamma^{v} \gamma^{\mu}\right)$ and $S[\ldots]$ denotes the symmetrisation of the Lorentz indices.

The operator in Eq. (4.1) is related to the transversity distribution $\Delta_{T} f_{k}$ which appears in the transversity structure function $h_{1}\left(x, Q^{2}\right)$ [75]. It can be measured in semi-inclusive deep-inelastic scattering [76] and polarised Drell-Yan processes [77]. The massive OME of this operator is de-

noted by $A_{q q, Q}^{\mathrm{NS}, \mathrm{TR},(3)}$ and involves the same diagrams as the vector non-singlet operator $A_{q q, Q}^{\mathrm{NS},(3)}$. Of course, different Feynman rules and a different projector have to be used, but the required master integrals turn out to be the same. Our calculation allows to extract the $N_{F}$-dependent parts of the anomalous dimensions of the transversity operator up to 3-loop order. The result for the anomalous dimensions and the complete $\mathrm{OME} A_{q q, Q}^{\mathrm{NS}, \mathrm{TR},(3)}$ are given in [59]. In principle the heavy flavour Wilson coefficients could be constructed in a similar fashion as for the other structure functions, but at this point the light flavour Wilson coefficients have not been calculated to a sufficient order yet.

\section{Conclusions}

Up to now, seven out of eight 3-loop massive operator matrix elements have been calculated analytically for general values of the Mellin variable $N$. Here, we discussed both the framework of calculation as well as a selected number of applications. The analytic calculation of the required 3-loop master integrals with additional local operator insertions led to the development and improvement of computer-algebraic and mathematical methods and tools. The packages Sigma, HarmonicSums, EvaluateMultiSums, SumProduction and SolveCoupledSystem both enabled the calculation and have benefited greatly from the challenges posed by the tasks arising from the project.

The largest contribution to the heavy flavour corrections to the structure function $F_{2}\left(x, Q^{2}\right)$ is expected to come from the Wilson coefficient $H_{g, 2}^{\mathrm{S}}$, which is not yet known due to the missing OME $A_{Q g}^{(3)}$. Nonetheless, a first impression of the impact of the heavy flavour corrections can be seen from our illustrations in Section 3. We also discussed the influence of the massive $\mathrm{OME} A_{q q, Q}^{\mathrm{NS},(3)}$ on the non-singlet polarised and charged-current structure functions $g_{1}\left(x, Q^{2}\right)$ and $x F_{3}\left(x, Q^{2}\right)$, which is of the order of a few percent in both cases. Due to the vanishing first moment of the nonsinglet OME, the polarised Bjorken and Gross-Llewellyn-Smith sum rules are only modified by shifting $N_{F}$ to $N_{F}+1$ if compared to the purely massless case with $N_{F}$ quark flavours in the region $Q^{2} \gg m^{2}$. Finally, we also calculated the massive OME for the non-singlet transversity operator, which enabled us to obtain the $N_{F}$-dependent parts of the 3-loop anomalous dimension of this operator. 


\section{References}

[1] S. Bethke et al., Workshop on Precision Measurements of $\alpha_{s}, 1110.0016$;

S. Moch et al., High precision fundamental constants at the TeV scale, 1405.4781 ;

S. Alekhin, J. Bümlein and S. O. Moch, $\alpha_{s}$ from global fits of parton distribution functions, Mod. Phys. Lett. A31 (2016) 1630023.

[2] S. Alekhin, J. Blümlein and S. Moch, The ABM parton distributions tuned to LHC data, Phys. Rev. D89 (2014) 054028, [1310.3059].

[3] P. Jimenez-Delgado and E. Reya, Delineating parton distributions and the strong coupling, Phys. Rev. D89 (2014) 074049, [1403.1852];

S. Dulat, T.-J. Hou, J. Gao, M. Guzzi, J. Huston, P. Nadolsky et al., New parton distribution functions from a global analysis of quantum chromodynamics, Phys. Rev. D93 (2016) 033006, [1506. 07443 ]; L. A. Harland-Lang, A. D. Martin, P. Motylinski and R. S. Thorne, Parton distributions in the LHC era: MMHT 2014 PDFs, Eur. Phys. J. C75 (2015) 204, [1412 . 3989 ];

NNPDF collaboration, R. D. Ball et al., Parton distributions for the LHC Run II, JHEP 04 (2015) 040, [1410.8849];

ZEUS, H1 collaboration, H. Abramowicz et al., Combination of measurements of inclusive deep inelastic $e^{ \pm} p$ scattering cross sections and QCD analysis of HERA data, Eur. Phys. J. $\mathbf{C 7 5}$ (2015) 580, [1506.06042].

[4] A. Accardi et al., A Critical Appraisal and Evaluation of Modern PDFs, Eur. Phys. J. C76 (2016) 471, [1603.08906].

[5] S. Alekhin, J. Blümlein, K. Daum, K. Lipka and S. Moch, Precise charm-quark mass from deep-inelastic scattering, Phys. Lett. B720 (2013) 172-176, [1212 . 2355].

[6] S. Alekhin, J. Blümlein, S.-O. Moch and R. Plačakytè, The new ABMP16 PDF, PoS DIS2016 (2016) 016, [1609.03327].

[7] M. Buza, Y. Matiounine, J. Smith, R. Migneron and W. L. van Neerven, Heavy quark coefficient functions at asymptotic values $Q^{2} \gg m^{2}$, Nucl. Phys. B472 (1996) 611-658, [hep-ph/9601302].

[8] A. J. Buras, Asymptotic Freedom in Deep Inelastic Processes in the Leading Order and Beyond, Rev. Mod. Phys. 52 (1980) 199;

E. Reya, Perturbative Quantum Chromodynamics, Phys. Rept. 69 (1981) 195;

J. Blümlein, The Theory of Deeply Inelastic Scattering, Prog. Part. Nucl. Phys. 69 (2013) 28-84, [1208.6087].

[9] J. Ablinger, J. Blümlein, S. Klein, C. Schneider and F. Wißbrock, 3-Loop Heavy Flavor Corrections to DIS with two Massive Fermion Lines, in 19th International Workshop on Deep-Inelastic Scattering and Related Subjects (DIS 2011) Newport News, Virginia, April 11-15, 2011, 2011. 1106.5937 ; J. Ablinger, J. Blümlein, A. Hasselhuhn, S. Klein, C. Schneider and F. Wißbrock, New Heavy Flavor Contributions to the DIS Structure Function $F_{2}\left(x, Q^{2}\right)$ at $\mathscr{O}\left(\alpha_{s}^{3}\right), P o S$ RADCOR2011 (2011) 031, [1202.2700];

J. Ablinger, J. Blümlein, A. De Freitas, A. Hasselhuhn, C. Schneider and F. Wißbrock. DESY-14-019.

[10] I. Bierenbaum, J. Blümlein and S. Klein, Mellin Moments of the $O\left(\alpha_{s}^{3}\right)$ Heavy Flavor Contributions to unpolarized Deep-Inelastic Scattering at $Q^{2} \gg m^{2}$ and Anomalous Dimensions, Nucl. Phys. B820 (2009) 417-482, [0904.3563].

[11] A. Behring, I. Bierenbaum, J. Blümlein, A. De Freitas, S. Klein and F. Wißbrock, The logarithmic contributions to the $O\left(\alpha_{s}^{3}\right)$ asymptotic massive Wilson coefficients and operator matrix elements in deeply inelastic scattering, Eur. Phys. J. C74 (2014) 3033, [1 403 . 6356]. 
[12] J. A. M. Vermaseren, A. Vogt and S. Moch, The third-order QCD corrections to deep-inelastic scattering by photon exchange, Nucl. Phys. B724 (2005) 3-182, [hep-ph / 0504242 ].

[13] E. Laenen, S. Riemersma, J. Smith and W. L. van Neerven, Complete $O\left(\alpha_{s}\right)$ corrections to heavy flavor structure functions in electroproduction, Nucl. Phys. B392 (1993) 162-228;

E. Laenen, S. Riemersma, J. Smith and W. L. van Neerven, $O\left(\alpha_{s}\right)$ corrections to heavy flavor inclusive distributions in electroproduction, Nucl. Phys. B392 (1993) 229-250;

S. Riemersma, J. Smith and W. L. van Neerven, Rates for inclusive deep inelastic electroproduction of charm quarks at HERA, Phys. Lett. B347 (1995) 143-151, [hep-ph/9411431].

[14] S. I. Alekhin and J. Blümlein, Mellin representation for the heavy flavor contributions to deep inelastic structure functions, Phys. Lett. B594 (2004) 299-307, [hep-ph / 0404034 ].

[15] M. Buza, Y. Matiounine, J. Smith and W. L. van Neerven, Charm electroproduction viewed in the variable flavor number scheme versus fixed order perturbation theory, Eur. Phys. J. C1 (1998) 301-320, [hep-ph/9612398].

[16] M. Buza, Y. Matiounine, J. Smith and W. L. van Neerven, $O\left(\alpha_{s}^{2}\right)$ corrections to polarized heavy flavor production at $Q^{2} \gg m^{2}$, Nucl. Phys. B485 (1997) 420-456, [hep-ph/9608342].

[17] I. Bierenbaum, J. Blümlein and S. Klein, Two-Loop Massive Operator Matrix Elements and Unpolarized Heavy Flavor Production at Asymptotic Values $Q^{2} \gg m^{2}$, Nucl. Phys. B780 (2007) 40-75, [hep-ph/0703285];

I. Bierenbaum, J. Blümlein, S. Klein and C. Schneider, Two-Loop Massive Operator Matrix Elements for Unpolarized Heavy Flavor Production to O(E), Nucl. Phys. B803 (2008) 1-41, [0803. 0273 ]; I. Bierenbaum, J. Blümlein and S. Klein, The Gluonic Operator Matrix Elements at $O\left(\alpha_{s}^{2}\right)$ for DIS Heavy Flavor Production, Phys. Lett. B672 (2009) 401-406, [0 901 . 0669 ].

[18] G. 't Hooft and M. J. G. Veltman, Regularization and Renormalization of Gauge Fields, Nucl. Phys. B44 (1972) 189-213;

J. F. Ashmore, A Method of Gauge Invariant Regularization, Lett. Nuovo Cim. 4 (1972) 289-290;

G. M. Cicuta and E. Montaldi, Analytic renormalization via continuous space dimension, Lett. Nuovo Cim. 4 (1972) 329-332;

C. G. Bollini and J. J. Giambiagi, Dimensional Renormalization: The Number of Dimensions as a Regularizing Parameter, Nuovo Cim. B12 (1972) 20-26.

[19] P. Nogueira, Automatic Feynman graph generation, J. Comput. Phys. 105 (1993) 279-289.

[20] J. A. M. Vermaseren, New features of FORM, math-ph/0010025;

M. Tentyukov and J. A. M. Vermaseren, The Multithreaded version of FORM, Comput. Phys. Commun. 181 (2010) 1419-1427, [hep-ph/0 702279];

J. Kuipers, T. Ueda, J. A. M. Vermaseren and J. Vollinga, FORM version 4.0, Comput. Phys. Commun. 184 (2013) 1453-1467, [1203.6543].

[21] T. van Ritbergen, A. N. Schellekens and J. A. M. Vermaseren, Group theory factors for Feynman diagrams, Int. J. Mod. Phys. A14 (1999) 41-96, [hep-ph/9802376].

[22] J. L. Lagrange, Nouvelle Recherches sur la nature et la Propagation du son, Miscellanea Taurinensia II (1760-61) 11-172, reprinted in Serret, J. A. (ed.), Oeuvres de Lagrange, vol. 1, pp. 151-316. Gauthier-Villars, Paris, 1867;

C. F. Gauß, Theoria attactionis corporum sphaeroidicorum ellipticorum homogeneorum methodo novo tractate, Commentationes scietas scientiarum Gottingensis recentiores II (1813), reprinted in Werke, vol. 5, pp. 3-22. Göttingen, 1867;

G. Green, An Essay on the Application of Mathematical Analysis to the Theories of Electricity and 
Magnetism, reprinted in Ferrers, N. M. (ed.), Mathematical papers of the late George Green, pp. 1-115. Macmillan, London, 1871;

M. Ostrogradski, Note sur une intégrale qui se rencontre dans le calcul de l'attraction des sphérö̈des, Mem. Ac. Sci St. Peters. 1 (1831) 39-53.

[23] K. G. Chetyrkin, A. L. Kataev and F. V. Tkachov, New Approach to Evaluation of Multiloop Feynman Integrals: The Gegenbauer Polynomial x Space Technique, Nucl. Phys. B174 (1980) 345-377;

K. G. Chetyrkin and F. V. Tkachov, Integration by Parts: The Algorithm to Calculate beta Functions in 4 Loops, Nucl. Phys. B192 (1981) 159-204;

F. V. Tkachov, A Theorem on Analytical Calculability of Four Loop Renormalization Group Functions, Phys. Lett. B100 (1981) 65-68.

[24] C. Studerus, Reduze - Feynman Integral Reduction in C++, Comput. Phys. Commun. 181 (2010) 1293-1300, [0 912 .2546];

A. von Manteuffel and C. Studerus, Reduze 2 - Distributed Feynman Integral Reduction, 1201.4330 .

[25] C. W. Bauer, A. Frink and R. Kreckel, Introduction to the GiNaC framework for symbolic computation within the C++ programming language, J. Symb. Comput. 33 (2000) 1, [C s/ 0004015 ].

[26] R. H. Lewis, “Computer Algebra System Fermat.” http://home.bway.net/lewis.

[27] C. F. Gauß, Disquisitiones generales circa seriam infinitam $1+\frac{\alpha \beta}{1 \cdot \gamma} x+\frac{\alpha(\alpha+1) \beta(\beta+1)}{1 \cdot 2 \cdot \gamma(\gamma+1)} x x+e t c$, Commentationes societatis regiae scientarum Gottingensis recentiores (1812) 3-48;

E. E. Kummer, Über die hypergeometrische Reihe $1+\frac{\alpha \cdot \beta}{1 \cdot \gamma} x+\frac{\alpha(\alpha+1) \beta(\beta+1)}{1 \cdot 2 \cdot \gamma(\gamma+1)} x^{2}$ $+\frac{\alpha(\alpha+1)(\alpha+2) \beta(\beta+1)(\beta+2)}{1 \cdot 2 \cdot 3 \cdot \gamma(\gamma+1)(\gamma+2)} x^{3}+\ldots$, J. reine angew. Math. 15 (1836) 39-83, 127-172;

W. N. Bailey, Generalized Hypergeometric Series. No. 32 in Cambridge Tracts in Mathematics and Mathematical Physics. Cambridge University Press, London, 1935;

L. J. Slater, Generalized hypergeometric functions. Cambridge University Press, Cambridge, 1966; I. S. Gradstein and I. M. Ryshik, Tables of series, products and integrals. Verlag Harri Deutsch, Thun, 1981;

P. Appell and J. Kampé de Fériet, Fonctions hypergéométriques et hypersphériques: polynômes d'Hermite. Gauthier-Villars, Paris, 1926;

P. Appell, Sur les fonctions hypergéométriques de plusieurs variables, les polynômes d'Hermite et autres fonctions sphériques dans l'hyperespace. Gauthier-Villars, Paris, 1925;

J. Kampé De Fériet, La fonction hypergéométrique. Gauthier-Villars, Paris, 1937;

H. Exton, Multiple Hypergeometric Functions and Applications. Ellis Horwood, Chichester, 1976;

H. Exton, Handbook of hypergeometric integrals. Ellis Horwood, Chichester, 1978;

H. M. Srivastava and P. W. Karlsson, Multiple Gaussian hypergeometric series. Ellis Horwood, Chichester, 1985.

[28] C. Schneider, Symbolic Summation in Difference Fields. PhD thesis, RISC, J. Kepler University Linz, 2001.

[29] M. Karr, Summation in Finite Terms, J. ACM 28 (1981) 305-350;

C. Schneider, A Collection of Denominator Bounds to Solve Parameterized Linear Difference

Equations in $\Pi \Sigma$-Extensions, An. Univ. Timisoara Ser. Mat.-Inform. 42 (2004) 163-179;

C. Schneider, Solving Parameterized Linear Difference Equations in Terms of Indefinite Nested Sums and Products, J. Differ. Equations Appl. 11 (2005) 799-821;

C. Schneider, Degree Bounds to Find Polynomial Solutions of Parameterized Linear Difference

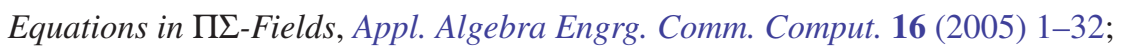

C. Schneider, Simplifying Sums in $\Pi \Sigma^{*}$-Extensions, J. Algebra Appl. 6 (2007) 415-441; 
C. Schneider, A Symbolic Summation Approach to Find Optimal Nested Sum Representations, in Motives, Quantum Field Theory, and Pseudodifferential Operators (A. Carey, D. Ellwood, S. Paycha and S. Rosenberg, eds.), vol. 12 of Clay Mathematics Proceedings, pp. 285-308, Amer. Math. Soc, 2010. 0904.2323 ;

C. Schneider, Parameterized Telescoping Proves Algebraic Independence of Sums, Ann. Comb. 14 (2010) 533-552, [0808.2596];

C. Schneider, Fast Algorithms for Refined Parameterized Telescoping in Difference Fields, in Computer Algebra and Polynomials, Applications of Algebra and Number Theory (J. Gutierrez, J. Schicho and M. Weimann, eds.), no. 8942 in Lecture Notes in Computer Science, pp. 157-191. Springer, 2015. 1307. 7887 ;

C. Schneider, A refined difference field theory for symbolic summation, J. Symbolic Comput. 43 (2008) 611-644, [0 808 .2543];

C. Schneider, A difference ring theory for symbolic summation, J. Symbolic Comput. 72 (2016) 82-127, [1408.2776];

C. Schneider, Summation Theory II: Characterizations of $R \Pi \Sigma$-extensions and algorithmic aspects, to appear in J. Symbolic Comput. (2016) , [1603.04285].

[30] C. Schneider, Symbolic Summation Assists Combinatorics, Sem. Lothar. Combin. 56 (2007) article $56 \mathrm{~b}$.

[31] C. Schneider, Simplifying Multiple Sums in Difference Fields, in Computer Algebra in Quantum Field Theory: Integration, Summation and Special Functions (C. Schneider and J. Blümlein, eds.), pp. 325-360. Springer, Vienna, 2013. 1304 .4134. DOI.

[32] J. Ablinger, J. Blümlein, S. Klein and C. Schneider, Modern Summation Methods and the Computation of 2- and 3-loop Feynman Diagrams, Nucl. Phys. Proc. Suppl. 205-206 (2010) 110-115, [1006.4797];

J. Blümlein, A. Hasselhuhn and C. Schneider, Evaluation of Multi-Sums for Large Scale Problems, PoS RADCOR2011 (2011) 032, [1202 . 4303];

C. Schneider, Symbolic Summation in Difference Fields and Its Application in Particle Physics, Computer Algebra Rundbrief 53 (2013) 8-12;

C. Schneider, Modern Summation Methods for Loop Integrals in Quantum Field Theory: The Packages Sigma, EvaluateMultiSums and SumProduction, J. Phys. Conf. Ser. 523 (2014) 012037, [1310.0160].

[33] J. Ablinger, A Computer Algebra Toolbox for Harmonic Sums Related to Particle Physics, Diploma thesis, J. Kepler University Linz, 2009.

[34] J. Ablinger, Computer Algebra Algorithms for Special Functions in Particle Physics. PhD thesis, J. Kepler University Linz, 2012. 1305.0687.

[35] J. Ablinger, J. Blümlein and C. Schneider, Harmonic Sums and Polylogarithms Generated by Cyclotomic Polynomials, J. Math. Phys. 52 (2011) 102301, [1105.6063].

[36] J. Ablinger, J. Blümlein and C. Schneider, Analytic and Algorithmic Aspects of Generalized Harmonic Sums and Polylogarithms, J. Math. Phys. 54 (2013) 082301, [1302 . 0378].

[37] J. Ablinger, J. Blümlein, C. G. Raab and C. Schneider, Iterated Binomial Sums and their Associated Iterated Integrals, J. Math. Phys. 55 (2014) 112301, [1407. 1822].

[38] J. Ablinger, The package HarmonicSums: Computer Algebra and Analytic aspects of Nested Sums, PoS LL2014 (2014) 019, [1 407 . 6180]. 
[39] H. Mellin, Om definita integraler, hvilka för obegränsadt växande värden af vissa heltaliga parametrar hafva till gränser hypergeometriska funktioner af särskilda ordningar, Acta Societatis Scientiarum Fennicae XX (1885) 1-39;

E. W. Barnes, A New Development of the Theory of the Hypergeometric Functions, Proc. London Math. Soc. s2-6 (1908) 141-177;

E. W. Barnes, A Transformation of Generalised Hypergeometric Series, Quart. J. Math. 41 (1910) $136-140$.

[40] V. A. Smirnov, Analytical result for dimensionally regularized massless on shell double box, Phys. Lett. B460 (1999) 397-404, [hep-ph/9905323];

J. B. Tausk, Nonplanar massless two loop Feynman diagrams with four on-shell legs, Phys. Lett. B469 (1999) 225-234, [hep-ph/9909506].

[41] V. A. Smirnov, Feynman integral calculus. Springer, Berlin, 2006;

M. Czakon, Automatized analytic continuation of Mellin-Barnes integrals, Comput. Phys. Commun. 175 (2006) 559-571, [hep-ph/0511200];

A. V. Smirnov and V. A. Smirnov, On the Resolution of Singularities of Multiple Mellin-Barnes Integrals, Eur. Phys. J. C62 (2009) 445-449, [0 901 . 0386 ].

[42] G. Almkvist and D. Zeilberger, The method of differentiating under the integral sign, J. Symb. Comp. 10 (1990) 571 - 591;

M. Apagodu and D. Zeilberger, Multi-variable Zeilberger and Almkvist-Zeilberger algorithms and the sharpening of Wilf-Zeilberger theory, Adv. Appl. Math. 37 (2006) 139 - 152.

[43] A. V. Kotikov, Differential equations method: New technique for massive Feynman diagrams calculation, Phys. Lett. B254 (1991) 158-164;

M. Caffo, H. Czyż, S. Laporta and E. Remiddi, Master equations for master amplitudes, Acta Phys. Polon. B29 (1998) 2627-2635, [hep-th/9807119];

M. Caffo, H. Czyż, S. Laporta and E. Remiddi, The Master differential equations for the two loop sunrise selfmass amplitudes, Nuovo Cim. A111 (1998) 365-389, [hep-th/9805118];

T. Gehrmann and E. Remiddi, Differential equations for two loop four point functions, Nucl. Phys. B580 (2000) 485-518, [hep-ph/9912329].

[44] J. Ablinger, A. Behring, J. Blümlein, A. De Freitas, A. von Manteuffel and C. Schneider, Calculating Three Loop Ladder and V-Topologies for Massive Operator Matrix Elements by Computer Algebra, Comput. Phys. Commun. 202 (2016) 33-112, [1509.08324].

[45] B. Zürcher, Rationale Normalformen von pseudo-linearen Abbildungen, Diploma thesis, ETH Zürich, 1994.

[46] S. Gerhold, Uncoupling Systems of Linear Ore Operator Equations, Diploma thesis, RISC, J. Kepler University Linz, 2002.

[47] J. Ablinger, J. Bluemlein, A. de Freitas and C. Schneider, A toolbox to solve coupled systems of differential and difference equations, PoS RADCOR2015 (2015) 060, [1601.01856];

J. Ablinger, A. Behring, J. Bluemlein, A. de Freitas and C. Schneider, Algorithms to solve coupled systems of differential equations in terms of power series, PoS LL2016 (2016) 005, [1608 05376 ].

[48] J. Ablinger, J. Blümlein, S. Klein, C. Schneider and F. Wißbrock, The $O\left(\alpha_{s}^{3}\right)$ Massive Operator Matrix Elements of $O\left(n_{f}\right)$ for the Structure Function $F_{2}\left(x, Q^{2}\right)$ and Transversity, Nucl. Phys. B844 (2011) 26-54, [1008.3347].

[49] J. Blümlein, A. Hasselhuhn, S. Klein and C. Schneider, The $O\left(\alpha_{s}^{3} n_{f} T_{F}^{2} C_{A, F}\right)$ Contributions to the Gluonic Massive Operator Matrix Elements, Nucl. Phys. B866 (2013) 196-211, [1205.4184]. 
[50] J. Ablinger, J. Blümlein, A. Hasselhuhn, S. Klein, C. Schneider and F. Wißbrock, Massive 3-loop Ladder Diagrams for Quarkonic Local Operator Matrix Elements, Nucl. Phys. B864 (2012) 52-84, [1206.2252].

[51] J. Ablinger, J. Blümlein, A. De Freitas, A. Hasselhuhn, A. von Manteuffel, M. Round et al., The Transition Matrix Element $A_{g q}(N)$ of the Variable Flavor Number Scheme at $O\left(\alpha_{s}^{3}\right)$, Nucl. Phys. B882 (2014) 263-288, [1 402 . 0359].

[52] J. Ablinger, J. Blümlein, A. De Freitas, A. Hasselhuhn, A. von Manteuffel, M. Round et al., The $O\left(\alpha_{s}^{3} T_{F}^{2}\right)$ Contributions to the Gluonic Operator Matrix Element, Nucl. Phys. B885 (2014) 280-317, [1405.4259].

[53] J. Ablinger, J. Blümlein, C. Raab, C. Schneider and F. Wißbrock, Calculating Massive 3-loop Graphs for Operator Matrix Elements by the Method of Hyperlogarithms, Nucl. Phys. B885 (2014) 409-447, [1403.1137].

[54] J. A. M. Vermaseren, Harmonic sums, Mellin transforms and integrals, Int. J. Mod. Phys. A14 (1999) 2037-2076, [hep-ph/9806280];

J. Blümlein and S. Kurth, Harmonic sums and Mellin transforms up to two loop order, Phys. Rev. D60 (1999) 014018, [hep-ph/9810241];

J. Blümlein, Analytic continuation of Mellin transforms up to two loop order, Comput. Phys.

Commun. 133 (2000) 76-104, [hep-ph / 0003100 ];

J. Blümlein, Algebraic relations between harmonic sums and associated quantities, Comput. Phys. Commun. 159 (2004) 19-54, [hep-ph / 0311046 ];

J. Blümlein, Structural Relations of Harmonic Sums and Mellin Transforms up to Weight $w=5$, Comput. Phys. Commun. 180 (2009) 2218-2249, [0 901 . 3106];

J. Blümlein, Structural Relations of Harmonic Sums and Mellin Transforms at Weight w=6, in Motives, Quantum Field Theory, and Pseudodifferential Operators (A. Carey, D. Ellwood, S. Paycha and S. Rosenberg, eds.), vol. 12 of Clay Mathematics Proceedings, p. 167, Amer. Math. Soc, 2010. 0901.0837.

[55] S. Moch, P. Uwer and S. Weinzierl, Nested sums, expansion of transcendental functions and multiscale multiloop integrals, J. Math. Phys. 43 (2002) 3363-3386, [hep-ph/ 0110083 ].

[56] J. Fleischer, A. V. Kotikov and O. L. Veretin, Analytic two loop results for selfenergy type and vertex type diagrams with one nonzero mass, Nucl. Phys. B547 (1999) 343-374, [hep-ph/9808242];

A. I. Davydychev and M. Y. Kalmykov, Massive Feynman diagrams and inverse binomial sums, Nucl. Phys. B699 (2004) 3-64, [hep-th/ 0303162 ];

S. Weinzierl, Expansion around half integer values, binomial sums and inverse binomial sums, J. Math. Phys. 45 (2004) 2656-2673, [hep-ph/ 0402131$].$

[57] E. Remiddi and J. A. M. Vermaseren, Harmonic polylogarithms, Int. J. Mod. Phys. A15 (2000) 725-754, [hep-ph/9905237].

[58] J. Ablinger, A. Behring, J. Blümlein, A. De Freitas, A. von Manteuffel and C. Schneider, The 3-loop pure singlet heavy flavor contributions to the structure function $F_{2}\left(x, Q^{2}\right)$ and the anomalous dimension, Nucl. Phys. B890 (2014) 48-151, [1409.1135].

[59] J. Ablinger, A. Behring, J. Blümlein, A. De Freitas, A. Hasselhuhn, A. von Manteuffel et al., The 3-Loop Non-Singlet Heavy Flavor Contributions and Anomalous Dimensions for the Structure Function $F_{2}\left(x, Q^{2}\right)$ and Transversity, Nucl. Phys. B886 (2014) 733-823, [1 406.4654 ].

[60] J. Ablinger et al. DESY-15-112. 
[61] J. Blümlein and H. Böttcher, QCD Analysis of Polarized Deep Inelastic Scattering Data, Nucl. Phys. B841 (2010) 205-230, [1 005 . 3113].

[62] A. Behring, J. Blümlein, A. De Freitas, A. von Manteuffel and C. Schneider, The 3-Loop Non-Singlet Heavy Flavor Contributions to the Structure Function $g_{1}\left(x, Q^{2}\right)$ at Large Momentum Transfer, Nucl. Phys. B897 (2015) 612-644, [1504.08217].

[63] D. Boer et al., Gluons and the quark sea at high energies: Distributions, polarization, tomography, 1108.1713 ;

A. Accardi et al., Electron Ion Collider: The Next QCD Frontier - Understanding the glue that binds us all, 1212.1701;

LHeC Study GRoup collaboration, J. L. Abelleira Fernandez et al., A Large Hadron Electron Collider at CERN: Report on the Physics and Design Concepts for Machine and Detector, J. Phys. G39 (2012) 075001, [1206.2913].

[64] S. Wandzura and F. Wilczek, Sum Rules for Spin Dependent Electroproduction: Test of Relativistic Constituent Quarks, Phys. Lett. B72 (1977) 195.

[65] J. D. Bjorken, Inelastic Scattering of Polarized Leptons from Polarized Nucleons, Phys. Rev. D1 (1970) 1376-1379.

[66] P. A. Baikov, K. G. Chetyrkin and J. H. Kühn, Adler Function, Bjorken Sum Rule, and the Crewther Relation to Order $\alpha_{s}^{4}$ in a General Gauge Theory, Phys. Rev. Lett. 104 (2010) 132004, [1001.3606].

[67] S. A. Larin, The singlet contribution to the Bjorken sum rule for polarized deep inelastic scattering, Phys. Lett. B723 (2013) 348-350, [1303 . 4021].

[68] P. A. Baikov, K. G. Chetyrkin and J. H. Kühn, Massless Propagators, R(s) and Multiloop QCD, Nucl. Part. Phys. Proc. 261-262 (2015) 3-18, [1501.06739].

[69] J. Blümlein, G. Falcioni and A. De Freitas, The Complete $O\left(\alpha_{s}^{2}\right)$ Non-Singlet Heavy Flavor Corrections to the Structure Functions $g_{1,2}^{e p}\left(x, Q^{2}\right), F_{1,2, L}^{e p}\left(x, Q^{2}\right), F_{1,2,3}^{v(\bar{v})}\left(x, Q^{2}\right)$ and the Associated Sum Rules, Nucl. Phys. B910 (2016) 568-617, [1605.05541].

[70] A. Behring, J. Blümlein, A. De Freitas, A. Hasselhuhn, A. von Manteuffel and C. Schneider, $O\left(\alpha_{s}^{3}\right)$ heavy flavor contributions to the charged current structure function $x F_{3}\left(x, Q^{2}\right)$ at large momentum transfer, Phys. Rev. D92 (2015) 114005, [1508.01449].

[71] J. Blümlein, A. Hasselhuhn, P. Kovačíková and S. Moch, $O\left(\alpha_{s}\right)$ Heavy Flavor Corrections to Charged Current Deep-Inelastic Scattering in Mellin Space, Phys. Lett. B700 (2011) 294-304, [11 04.344 9]; J. Blümlein, A. Hasselhuhn and T. Pfoh, The $O\left(\alpha_{s}^{2}\right)$ heavy quark corrections to charged current deep-inelastic scattering at large virtualities, Nucl. Phys. B881 (2014) 1-41, [1401.4352].

[72] D. J. Gross and C. H. Llewellyn Smith, High-energy neutrino-nucleon scattering, current algebra and partons, Nucl. Phys. B14 (1969) 337-347.

[73] P. A. Baikov, K. G. Chetyrkin and J. H. Kühn, Adler Function, DIS sum rules and Crewther Relations, Nucl. Phys. Proc. Suppl. 205-206 (2010) 237-241, [1007 . 0478 ].

[74] P. A. Baikov, K. G. Chetyrkin, J. H. Kühn and J. Rittinger, Adler Function, Sum Rules and Crewther Relation of Order $O\left(\alpha_{s}^{4}\right)$ : the Singlet Case, Phys. Lett. B714 (2012) 62-65, [1206.1288].

[75] V. Barone, A. Drago and P. G. Ratcliffe, Transverse polarisation of quarks in hadrons, Phys. Rept. 359 (2002) 1-168, [hep-ph/ 0104283 ]. 
[76] J. P. Ralston and D. E. Soper, Production of Dimuons from High-Energy Polarized Proton Proton Collisions, Nucl. Phys. B152 (1979) 109;

R. L. Jaffe and X.-D. Ji, Chiral odd parton distributions and polarized Drell-Yan, Phys. Rev. Lett. 67 (1991) 552-555;

R. L. Jaffe and X.-D. Ji, Chiral odd parton distributions and Drell-Yan processes, Nucl. Phys. B375 (1992) 527-560;

J. L. Cortes, B. Pire and J. P. Ralston, Measuring the transverse polarization of quarks in the proton, Z. Phys. C55 (1992) 409-416.

[77] X. Artru and M. Mekhfi, Transversely Polarized Parton Densities, their Evolution and their Measurement, Z. Phys. C45 (1990) 669;

J. C. Collins, Fragmentation of transversely polarized quarks probed in transverse momentum distributions, Nucl. Phys. B396 (1993) 161-182, [hep-ph/9208213];

R. L. Jaffe and X.-D. Ji, Novel quark fragmentation functions and the nucleon's transversity distribution, Phys. Rev. Lett. 71 (1993) 2547-2550, [hep-ph/9307329];

R. D. Tangerman and P. J. Mulders, Polarized twist-three distributions $g_{T}$ and $h_{L}$ and the role of intrinsic transverse momentum, hep-ph/9408305;

D. Boer and P. J. Mulders, Time reversal odd distribution functions in leptoproduction, Phys. Rev. D57 (1998) 5780-5786, [hep-ph/9711485]. 